\title{
Event-Driven Simulation of Power Electronics in the Complementarity Systems Framework
}

\author{
Jeroen Tant and Johan Driesen \\ Department of Electrical Engineering (ESAT) \\ Research Group ELECTA \\ KU Leuven \\ Leuven, Belgium \\ Email: jeroen.tant@esat.kuleuven.be
}

\author{
Wim Michiels \\ Department of Computer Science \\ Scientific Computing Research Group \\ KU Leuven \\ Leuven, Belgium
}

\begin{abstract}
A new event-driven method is proposed for the numerical simulation of power electronic circuits with ideal switches and diodes, and their accompanying control systems. The power electronic circuit is modeled as a complementarity system and the control system is modeled separately. It is shown that the diode state determination problem can be formulated as a complementarity problem. Discontinuous jumps in the state variables are allowed after a switching event. The method is applied to two examples and the numerical accuracy with respect to the step size is compared with time-stepping methods. The importance of the event localization accuracy is discussed.
\end{abstract}

\section{INTRODUCTION}

Conventional time domain analog circuit simulation methods (e.g. solvers in the Spice family) are in some cases impractical for simulations of power electronic systems at a large time scale [1], [2]. Non-smooth methods allow discontinuities in the time signals, and model the circuit with ideal or piece-wise linear switches and diodes to speed up the simulation. Numerical simulation methods for the non-smooth approach can be classified into event-driven methods [2] [8] and time-stepping methods [9]-[11]. The available timestepping methods do not need an event localization algorithm, but have a low order of accuracy with respect to the step size.

Recently, the potential of the complementarity system formalism [12], [13] for the modeling of power electronic circuits has been extensively demonstrated [11], [14]-[16]. Numerical methods in the context of power electronic circuits that make use of the complementarity formalism, are currently mainly based on time-stepping methods with a low order backward Euler integration scheme [10], [11], [15], [17]. It is difficult to incorporate the various control systems used in practical applications in the complementarity system model. This is currently only possible for analog controllers based on a comparator [10], [11], [16].

In this paper, an event-driven method is proposed for the simulation of power electronic systems. Only the circuit is modeled as a complementarity system, and a separate model is used for the control system. There are no limitations on the type of control system. The event-driven method makes it possible to use integration methods with a higher order than the backward Euler time-stepping method.
The problem of determining state variables and diode states after a switching event is formulated as a complementarity problem. The main advantage compared to other diode state determination algorithms [2]-[7], is that complementarity problems are a well-known class of problems in the field of numerical optimization, with existing efficient solution methods for the linear and nonlinear case [18]. Furthermore, discontinuous jumps of state variables are allowed. This facilitates the simulation of switched capacitor circuits that have been modeled with ideal capacitors in order to reach an efficient simulation time.

\section{NOTATION}

Given an index set $\mathcal{I} \subseteq\{1,2, \ldots, n\}$, then $n_{\mathcal{I}}$ denotes cardinality of $\mathcal{I}$. Given a vector $x \in \mathbb{R}^{n}$, then the vector $x_{\mathcal{I}}$ contains the components of $x$ with indices in $\mathcal{I}$, and the vector $x_{\overline{\mathcal{I}}}$ the components of $x$ with indices that are not in $\mathcal{I}$. Similarly, given a matrix $A$ and index sets $\mathcal{I}$ and $\mathcal{J}$, then $A_{\mathcal{I}, \mathcal{J}}=\left(A_{i, j}\right)_{i \in \mathcal{I} ; j \in \mathcal{J}}$. Furthermore, the matrix $A_{\mathcal{I}}$ is formed by the rows of $A$ that are in $\mathcal{I}$, and $A_{\bullet_{\mathcal{J}}}$ by the columns of $A$ that are in $\mathcal{J}$.

\section{Complementarity Problem Classes}

In this section, a number of complementarity problem classes and solution methods are listed and summarized. The problem classes are well known from literature [9], [19], [20] and will be used throughout this paper.

\section{A. Linear Complementarity Problem}

The Linear Complementarity Problem (LCP) is defined as finding unknown vectors $w \in \mathbb{R}^{n}$ and $z \in \mathbb{R}^{n}$, which satisfy the equations:

$$
\begin{aligned}
& w=M z+q, \\
& 0 \leq w \perp z \geq 0,
\end{aligned}
$$

where $q \in \mathbb{R}^{n}$ is a known vector and $M \in \mathbb{R}^{n \times n}$ is a square matrix. The orthogonality operator $\perp$ enforces the element-wise complementarity condition on the vectors $z$ and $w: z_{i} w_{i}=0$ for all $i$. The LCP can be infeasible without solutions, or feasible with a unique solution or multiple solutions, dependent on the structure of $M$ [19]. Two main 
groups of algorithms to solve the LCP are pivoting methods (e.g. Lemke's algorithm), similar to the Gauss elimination method for linear systems, and interior point methods [20]. When $\mathrm{M}$ is positive-semidefinite, which will be the case for the circuit equations encountered in this paper, the LCP can be reformulated as a convex Quadratic Programming (QP) optimization problem [19]:

$$
\begin{array}{cl}
\underset{z}{\operatorname{minimize}} & \frac{1}{2} z^{T}\left(M+M^{T}\right) z+z^{T} q, \\
\text { subject to } & z \geq 0, M z+q \geq 0 .
\end{array}
$$

A solution of the QP in (2) is also a solution of the corresponding LCP when the objective function is equal to zero, otherwise the LCP is infeasible. The solution is unique when $M$ is positive-definite. An infinite number of solutions can exist when $M$ is positive-semidefinite, and the set of all possible solutions is convex [21].

\section{B. Mixed Linear Complementarity Problem}

An extension of the LCP in (1) is the Mixed Linear Complementarity Problem (MLCP):

$$
\begin{aligned}
0 & =A x+B z+b, \\
w & =C x+M z+q, \\
0 & \leq w \perp z \geq 0,
\end{aligned}
$$

where $x \in \mathbb{R}^{m}$ is an unknown vector and $b \in \mathbb{R}^{m}$ is a known vector. The MLCP combines an LCP with a linear system of equations. The LCP algorithms discussed in section III-A, with some modifications, are also applicable to MLCPs.

\section{Nonlinear Complementarity Problem}

The nonlinear variant of the LCP in (1) is the Nonlinear Complementarity Problem (NCP):

$$
\begin{aligned}
& w=F(z), \\
& 0 \leq w \perp z \geq 0,
\end{aligned}
$$

where $F: \mathbb{R}^{n} \rightarrow \mathbb{R}^{n}$ is a nonlinear mapping.

\section{Mixed Complementarity Problem}

The nonlinear variant of the MLCP is the Mixed Complementarity Problem (MCP):

$$
\begin{aligned}
0 & =G(z, x), \\
w & =F(z, x), \\
0 & \leq w \perp z \geq 0,
\end{aligned}
$$

where $G: \mathbb{R}^{n} \times \mathbb{R}^{m} \rightarrow \mathbb{R}^{m}$ and $F: \mathbb{R}^{n} \times \mathbb{R}^{m} \rightarrow \mathbb{R}^{n}$ are nonlinear mappings.

A comparison of various MCP algorithms is given in [22]. One popular solver is the PATH solver [23]. MCP algorithms are also applicable to the NCP, as the NCP is a subclass of the MCP.

\section{E. Complementarity Systems}

A semi-explicit complementarity system has the form [12]:

$$
\begin{aligned}
\dot{x} & =F(x, z, t), \\
w & =G(x, z, t), \\
0 & \leq w \perp z \geq 0,
\end{aligned}
$$

where $x \in \mathbb{R}^{n}$ is the state variable vector, $z \in \mathbb{R}^{m}$ and $w \in$ $\mathbb{R}^{m}$ are complementarity variable vectors.

\section{MODELING}

The power electronic system model is split into a circuit model and a control system model.

\section{A. Circuit Model}

The topology of the power electronic circuit is associated with a connected directed graph. A branch-oriented circuit modeling method is applied [24], in order to obtain the circuit equations in terms of branch currents $i$ and branch voltages $v$.

The index set of all branches $\mathcal{B}$ is divided into a set with capacitors $\mathcal{C}$, inductors $\mathcal{L}$, resistors $\mathcal{R}$, state controllable voltage sources $\mathcal{U}$, state controllable current sources $\mathcal{J}$, ideal switches $\mathcal{S}$ and ideal diodes $\mathcal{D}$. The switch index set $\mathcal{S}$ is further divided into a set with open switches $\mathcal{S}_{0}$ and closed switches $\mathcal{S}_{1}$ The constitutive equations for the circuit components are given by

$$
\begin{aligned}
C \dot{v}_{\mathcal{C}} & =i_{\mathcal{C}}, \\
L \dot{i}_{\mathcal{L}} & =v_{\mathcal{L}}, \\
R i_{\mathcal{R}} & =v_{\mathcal{R}}, \\
v_{\mathcal{U}} & =f_{\mathcal{U}}\left(v_{\mathcal{C}}, i_{\mathcal{L}}, t\right), \\
i_{\mathcal{J}} & =f_{\mathcal{J}}\left(v_{\mathcal{C}}, i_{\mathcal{L}}, t\right), \\
0 i_{\mathcal{D}} & \perp-v_{\mathcal{D}} \geq 0, \\
i_{\mathcal{S}_{0}} & =0, \\
v_{\mathcal{S}_{1}} & =0,
\end{aligned}
$$

where $C$ is the diagonal capacitance matrix, $L$ is the symmetric inductance matrix assumed to be non-singular, and $R$ is the diagonal resistance matrix. The controllable voltage sources and current sources are only dependent on the state variables of the circuit.

It is assumed that the circuit is well-posed, therefore loops with only voltage sources and cut-sets with only current sources are forbidden. Furthermore, loops with capacitors only, or capacitors and voltage sources only, are broken with an additional switch without loss of generality. A fixed closed state is assigned to the switch afterwards. Similarly, cut-sets with inductors only, or inductors and current sources only, are bridged with an additional switch with a fixed open state [25].

Afterwards, a tree is identified in the circuit graph, connecting all nodes of the circuit, and containing all capacitors, no inductors, all voltage sources, and no current sources. It can be shown that it is possible to find such a tree under the assumptions defined in the above paragraph [24]. Branches in the tree are called twigs and the other branches are called links. Given an index set $\mathcal{I}$ with some branches of the circuit, then 
$\mathcal{I}_{t}$ denotes the set with branches in $\mathcal{I}$ that are twigs, and $\mathcal{I}_{l}$ denotes the set with branches in $\mathcal{I}$ that are links. Consequently $\mathcal{C}_{l}=\emptyset, \mathcal{C}_{t}=\mathcal{C}, \mathcal{U}_{l}=\emptyset, \mathcal{U}_{t}=\mathcal{U}, \mathcal{L}_{l}=\mathcal{L}, \mathcal{L}_{t}=\emptyset, \mathcal{J}_{l}=\mathcal{J}$, and $\mathcal{J}_{t}=\emptyset$.

Every twig defines a unique cut-set, and every link defines a unique loop. This leads to a fundamental cut-set matrix $Q \in$ $\mathbb{R}^{n_{t} \times n_{\mathcal{B}}}$ and a fundamental loop matrix $B \in \mathbb{R}^{n_{l} \times n_{\mathcal{B}}}$ in the following form:

$$
\begin{aligned}
& Q=\left(\begin{array}{ll}
I_{n_{t}}-F^{T}
\end{array}\right), \\
& B=\left(\begin{array}{ll}
F & I_{n_{l}}
\end{array}\right),
\end{aligned}
$$

where $F \in \mathbb{R}^{n_{l} \times n_{t}}=\left(f_{i, j}\right)$ is a matrix with $f_{i, j}=1$ if branch $j$ is in a loop with link $i$ with the same orientation, $f_{i, j}=-1$ if branch $j$ is in a loop with link $i$ with opposite orientation, and $f_{i, j}=0$ if branch $j$ is not in a loop with link $i$ [24].

The Kirchoff Current Laws (KCL) and Kirchoff Voltage Laws (VCL) can then be written as:

$$
\begin{aligned}
& (\mathrm{KCL}) \quad i_{t}=F^{T} i_{l}, \\
& (\mathrm{KVL}) \quad v_{l}=-F v_{t} .
\end{aligned}
$$

The system of equations formed by (7) and (9) describes the complete circuit.

The following notation is applied:

$$
x=\left(\begin{array}{c}
i_{\mathcal{L}} \\
v_{\mathcal{C}}
\end{array}\right), \quad w=\left(\begin{array}{c}
-v_{\mathcal{S}_{l}} \\
i_{\mathcal{S}_{t}} \\
-v_{\mathcal{D}_{l}} \\
i_{\mathcal{D}_{t}}
\end{array}\right), \quad z=\left(\begin{array}{c}
i_{\mathcal{S}_{l}} \\
-v_{\mathcal{S}_{t}} \\
i_{\mathcal{D}_{l}} \\
-v_{\mathcal{D}_{t}}
\end{array}\right),
$$

where $x$ are the circuit state variables, and $w$ and $z$ are the complementarity variables. After elimination of the the variables $i_{\mathcal{R}}$ and $v_{\mathcal{R}}$, the circuit equations are finally rewritten in complementarity system form:

$$
\begin{aligned}
\dot{x} & =A x+B z+f(x, t), \\
w & =C x+D z+g(x, t), \\
0 & \leq w_{i} \perp z_{i} \geq 0, \quad i \in \mathcal{D}, \\
\mathcal{K}_{s_{i}}^{*} & \ni w_{i} \perp z_{i} \in \mathcal{K}_{s_{i}}, \quad i \in \mathcal{S},
\end{aligned}
$$

where indices in $\mathcal{D}$ represent the diodes and the indices in $S$ represent the switches. The vector $s \in\{1,0\}^{m}$ denotes the current state of the switches. The cone $\mathcal{K}_{s_{i}}$ and dual cone $\mathcal{K}_{s_{i}}^{*}$ are dependent on $s$ :

$$
\mathcal{K}_{0}=\mathcal{K}_{1}^{*}=\{0\}, \quad \mathcal{K}_{1}=\mathcal{K}_{0}^{*}=\mathbb{R} .
$$

A switch state $s_{i}=1$ represents a closed switch when the switch is a link, and an open switch when the switch is a twig. The system in (11) is a cone complementarity system [15].

\section{B. Control System Model}

The control system model determines the switch state of controllable switches and triggers an event when a switch state changes. This model can be governed by measurements on the circuit variables. The control system can be modeled, for example, as a continuous-time dynamical system, a discretetime DSP algorithm, or an event-driven finite state machine.
There is no limitation on the type of the control system. However, some theoretical control systems, such as an ideal sliding mode controller, might result in an accumulation of events. This accumulation of events can be resolved by adding a hysteresis band to the controller as would be needed to implement the controller practically.

\section{Circuit Solution}

\section{A. Static Solution}

The static circuit problem is defined as finding all currents $i$ and voltages $v$ for a given consistent state vector $x$, a given consistent switch state vector $s$, and given consistent values for the current sources and voltage sources. This problem can be solved by solving the MLCP formed by equations (11b) to (11d). However, special care is needed when there is a loop with only capacitors combined with voltage sources (zero or more), switches (zero or more) and diodes with the same orientation (zero or more), or when there is a cut set with only inductors combined with current sources (zero or more), switches (zero or more) and diodes with the same orientation (zero or more). In this paper, they are called CVSD loops and LISD cut-sets respectively.

In case of a CVSD loop, there is one link diode or link switch that defines the loop. The equation for this link's voltage is extracted out of (11b) as

$$
w_{k}=C_{k \bullet} x+B_{k, \mathcal{H}} z_{\mathcal{H}}+g_{k}(x, t),
$$

where $k$ is the index of the link diode or link switch and $\mathcal{H}$ is the index set of other twig diodes and switches in the same loop. When all switches in the CVSD loop are closed, and when $C_{k} \bullet x+g_{k}(x, t)=0$, then the voltages of the diodes in the loop are zero as they have the same orientation: $w_{k}=$ $z_{\mathcal{H}}=0$. In this case, (13) becomes degenerate and should be replaced with the equation

$$
\dot{w}_{k}=C_{k \bullet} \dot{x}+B_{k, \mathcal{H}} \dot{z}_{\mathcal{H}}+\frac{\partial g_{k}}{\partial x}(x, t) \dot{x}+\frac{\partial g_{k}}{\partial t}(x, t),
$$

where $\dot{w}_{k}$ and $\dot{z}_{\mathcal{H}}$ are new unknown variables. The corresponding complementarity constraints in (11c)-(11d) should be altered to

$$
0 \leq \dot{w}_{k} \perp z_{k} \geq 0
$$

when $k$ corresponds with a diode,

$$
\dot{w}_{k}=0,
$$

when $\mathrm{k}$ is a switch, and

$$
\begin{gathered}
0 \leq w_{i} \perp \dot{z}_{i} \geq 0, \quad i \in \mathcal{D} \cap \mathcal{H}, \\
\mathcal{K}_{s_{i}}^{*} \ni w_{i} \perp \dot{z}_{i} \in \mathcal{K}_{s_{i}}, \quad i \in \mathcal{S} \cap \mathcal{H} .
\end{gathered}
$$

The resulting problem is still an MLCP, as $x$ is a given variable in (14), and $\dot{x}$ is linearly related to the unknown variables through (11a). The reasoning for LISD cut-sets is completely analogous.

Even when all CVSD loops and LISD cut-sets are resolved, the MLCP solutions are not necessarily unique. Multiple current paths can exists when there is a loop with closed switches and diodes only (SD loop). Likewise, the voltage 


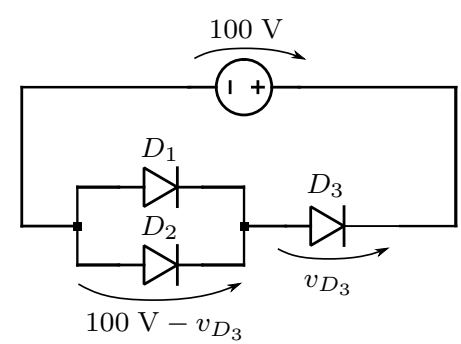

(a)

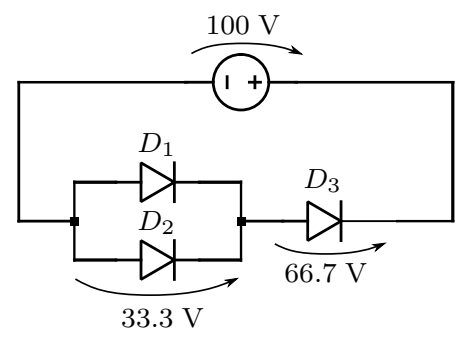

(b)

Fig. 1. Example circuit with multiple solutions (a) and the minimum norm solution (b).

across the elements in a cut-set with open switches and diodes only (SD cut-set) is not always uniquely defined. When the minimum norm solution is chosen, the free voltages and currents are distributed as if the non-ideal diodes and switches have an equal impedance. An example of a circuit with multiple solutions is given in Fig. 1a and the minimum norm solution is shown in Fig. 1b. Only diode and switch variables can have multiple solutions, $\dot{x}$ is thus uniquely defined after solving the MLCP.

\section{B. Diode State Determination}

It is possible that some diode states are still unknown after finding a minimum norm solution for the static MLCP problem defined in section V-A. This is the case when a diode's voltage and current are both zero $\left(z_{i}=w_{i}=0\right)$. The unknown diode states can be resolved by adding the following constraints to the circuit equations:

$$
\begin{array}{rlrl}
\left(w_{i}, \dot{w}_{i}, w_{i}^{(2)}, w_{i}^{(3)}, \ldots\right) & \succeq 0 & & i \in \mathcal{D}, \text { (16a) } \\
\left(z_{i}, \dot{z}_{i}, z_{i}^{(2)}, z_{i}^{(3)}, \ldots\right) & \succeq 0 & & i \in \mathcal{D}, \text { (16b) } \\
w_{i}^{(k)} & \perp z_{i}^{(j)} & \forall j, k \geq 0, \quad i \in \mathcal{D}, \text { (16c) } \\
w_{i}^{(j)} & \in \mathcal{K}_{s_{i}}^{*} & \forall j \geq 0, \quad i \in \mathcal{S},(16 \mathrm{~d}) \\
z_{i}^{(j)} & \in \mathcal{K}_{s_{i}} & \forall j \geq 0, \quad i \in \mathcal{S}, \quad(16 \mathrm{e})
\end{array}
$$

where the operator $\succeq$ denotes lexicographic nonnegativity [12], [14]. The resulting problem is known as the Dynamic Complementarity Problem (DCP) [12], [14].

The solution method described in [12] can be used to solve the DCP recursively. At each iteration $k$ of the DCP algorithm, the index set $\mathcal{J}$ represents diodes and switches that are unambiguously in the state $z_{\mathcal{J}}^{(j)}=0 \forall j$, and the index set

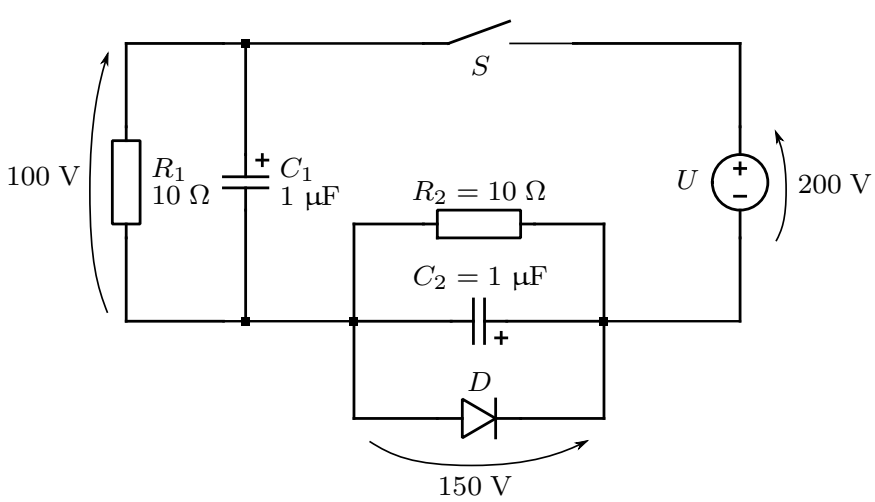

(a)

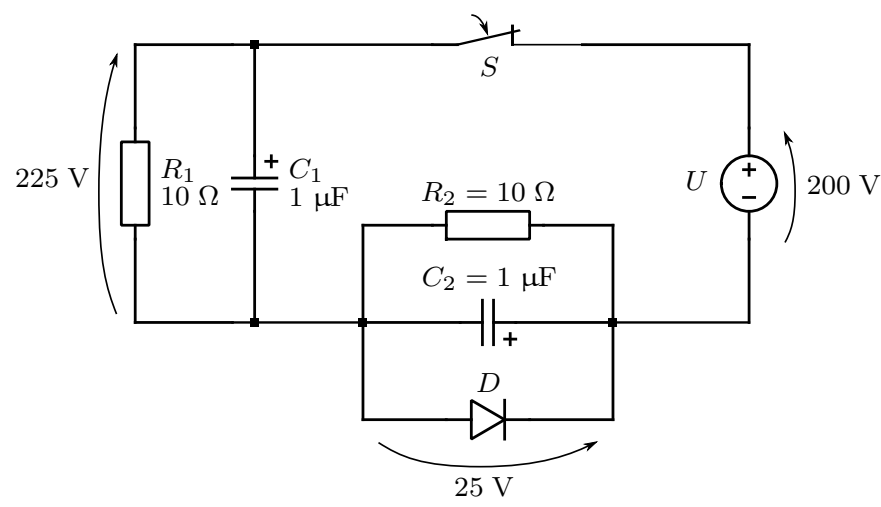

(b)

Fig. 2. Example circuit where the closing of switch $S$ induces a state jump. The capacitor voltages right before the event are shown in (a) and the voltages after the event are shown in (b).

$\mathcal{I}$ represents diodes and switches that are unambiguously in the state $w_{\mathcal{I}}^{(j)}=0 \forall j$. The index set $\mathcal{K}$ represents diodes with an unresolved state at iteration $k-1: z_{\mathcal{K}}^{(k-1)}=w_{\mathcal{K}}^{(k-1)}=0$. The following MLCP is solved at each iteration:

$$
\begin{aligned}
x^{(k)} & =A x^{(k-1)}+B_{\bullet \mathcal{I}} z_{\mathcal{I}}^{(k-1)}+f^{(k-1)}, \\
0 & =C_{\mathcal{I} \bullet} x^{(k)}+D_{\mathcal{I} \mathcal{K}} z_{\mathcal{K}}^{(k)}+D_{\mathcal{I} \mathcal{I}} z_{\mathcal{I}}^{(k)}+g_{\mathcal{I}}^{(k)}, \\
w_{\mathcal{K}}^{(k)} & =C_{\mathcal{K} \bullet} x^{(k)}+D_{\mathcal{K} \mathcal{K}} z_{\mathcal{K}}^{(k)}+D_{\mathcal{K} \mathcal{I}} z_{\mathcal{I}}^{(k)}+g_{\mathcal{K}}^{(k)}, \\
0 & \leq w_{\mathcal{K}}^{(k)} \perp z_{\mathcal{K}}^{(k)} \geq 0,
\end{aligned}
$$

where the derivatives $x^{(k)}, g^{(k)}$ and $f^{(k-1)}$ are only dependent on the solutions of previous iterations. When there is an index $i$ with $z_{i \in \mathcal{K}}^{(k)}>0$ or $w_{i \in \mathcal{K}}^{(k)}>0$, the corresponding diode state is resolved and $i$ can be added to $\mathcal{I}$ or $\mathcal{J}$ respectively. The algorithm continues until all diode states are resolved, or until it is clear that $z_{\mathcal{K}}^{(j)}=w_{\mathcal{K}}^{(j)}=0 \forall j$.

\section{State Reinitialization}

It is possible that a jump in the state variables occurs after an event where a switch in a CVSD loop closes, or when a switch in a LISD cut-set opens. An example is shown in Fig. 2.

State jump rules [25] can be incorporated to calculate the new state variables $x^{+}$after the event time $t$. The integral of Dirac impulses in the complementarity variables is given with 
the following notation:

$$
\delta z=\int_{t^{-}}^{t^{+}} z \mathrm{~d} t, \quad \delta w=\int_{t^{-}}^{t^{+}} w \mathrm{~d} t .
$$

The following set of equations is obtained after integration of the equations in (11):

$$
\begin{aligned}
x^{+}-x^{-} & =B \delta z \\
\delta w & =D \delta z, \\
0 & \leq \delta w_{i} \perp \delta z_{i} \geq 0, \quad i \in \mathcal{D} . \\
\mathcal{K}_{s_{i}}^{*} & \ni \delta w_{i} \perp \delta z_{i} \in \mathcal{K}_{s_{i}}, \quad i \in \mathcal{S} .
\end{aligned}
$$

These equations form a MLCP with multiple solutions. The set of possible solutions is convex under the assumption that $D$ is positive-semidefinite (see section III-A). As a result, at least one of the variables $\delta w_{i}$ or $\delta z_{i}$ is constrained to zero. Therefore, an index set $\mathcal{G}$ can be found for which $\delta w_{\mathcal{G}}=0$ and $\delta z_{\overline{\mathcal{G}}}=0$. The complementarity equations of (11) are added to (19) in order to find a unique solution for $x^{+}$. The resulting state reinitialization problem is defined as finding values for $x^{+}, z^{+}, w^{+}, \delta z_{\mathcal{G}}$ and $\delta w_{\overline{\mathcal{G}}}$ that satisfy the equations

$$
\begin{array}{rlrl}
x^{+} & =x^{-}+B_{\bullet \mathcal{G}} \delta z_{\mathcal{G}}, & & \\
\delta w_{\overline{\mathcal{G}}} & =D_{\overline{\mathcal{G}} \mathcal{G}} \delta z_{\mathcal{G}}, & & \\
w^{+} & =C x^{+}+D z^{+}+g\left(x^{+}, t^{+}\right), & \\
0 & \leq w_{i}^{+} \perp z_{i}^{+} \geq 0, & & i \in \mathcal{D}, \\
0 & \leq w_{i}^{+} \perp \delta z_{i} \geq 0, & & i \in \mathcal{D} \cap \mathcal{G}, \\
0 & \leq \delta w_{i} \perp z_{i}^{+} \geq 0, & & i \in \mathcal{D} \cap \overline{\mathcal{G}}, \\
\mathcal{K}_{s_{i}}^{*} & \ni w_{i}^{+} \perp z_{i}^{+} \in \mathcal{K}_{s_{i}}, & & i \in \mathcal{S} .
\end{array}
$$

This problem is nonlinear in $g\left(x^{+}, t^{+}\right)$, when there are nonlinear controllable voltage sources.

\section{Event-Driven Simulation Procedure}

The following five-step procedure is executed after the detection of an event at time $t$ :

1) The state of all switches at time $t$ is computed with the control system model.

2) The state variables are reinitialized after possible state jumps by solving the complementarity problem as described in section $\mathrm{V}-\mathrm{C}$.

3) The diode states are determined for the next time interval by solving the dynamic complementarity problem with the procedure of section V-B.

4) Smooth simulation of the circuit equations with a conventional integration method. The control system model is simulated in parallel with the circuit equations. The insertion of the determined diode states in (11) results in a semi-explicit Differential Algebraic Equation (DAE) form. Conventional DAE solvers can be used to simulate the circuit. An Ordinary Differential Equation (ODE) solver can be used when the complementarity variables $z$ and $w$ are eliminated.

5) Event detection and localization with a root-finding algorithm. The event detection algorithm should monitor the

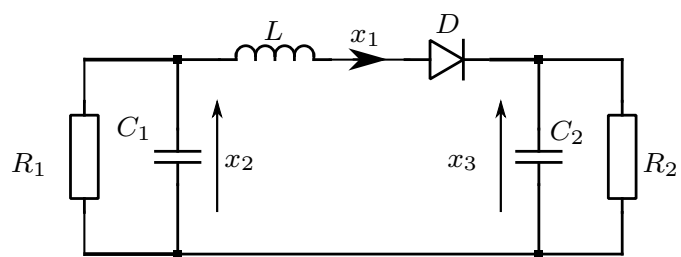

Fig. 3. Circuit diagram for numerical example 1: a simple diode circuit. Parameters: $L=10 \mathrm{mH}, C_{1}=C_{2}=100 \mu \mathrm{F}, R_{1}=200 \Omega, R_{2}=100 \Omega$, and $x\left(t_{0}\right)=\left(\begin{array}{lll}0 \mathrm{~A} & 100 \mathrm{~V} & 110 \mathrm{~V}\end{array}\right)^{T}$.

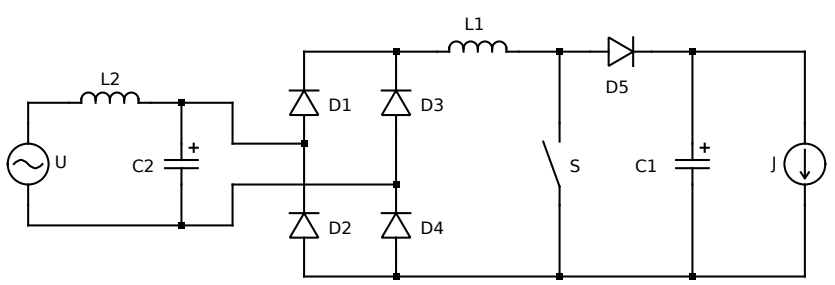

Fig. 4. Circuit diagram for numerical example 2: a peak-current controlled PFC. Parameters: $L_{1}=10 \mathrm{mH}, C_{1}=820 \mu \mathrm{F}, C_{2}=3.3 \mu \mathrm{F}$, $L_{1}=0.3 \mathrm{mH}, v_{U}=230 \sqrt{2} \sin (50 \pi t), i_{J}=\frac{800+50 \sin (400 \pi t)}{x_{2}}$, $x=\left(\begin{array}{llll}i_{L_{1}} & v_{C_{1}} & i_{L_{2}} & v_{C_{2}}\end{array}\right)^{T}, x\left(t_{0}\right)=\left(\begin{array}{lllll}0 \mathrm{~A} & 380 \mathrm{~V} & 0 \mathrm{~V} & 0 \mathrm{~A}\end{array}\right)^{T^{T}}$.

positiveness of $z_{\mathcal{D}}$ and $w_{\mathcal{D}}$. When a sign change is detected in one of these variables, the exact zero-crossing event time $t$ is localized with a root-finding algorithm. The control system model can also define zero-crossing test functions on measured variables. Furthermore, it is possible that control system triggers an event with an internal timer. In this case, event localization is not needed because the exact switching time is known.

\section{NumERICAL RESULTS}

Two examples are considered: a simple diode circuit in Fig. 3 and a peak-current controlled PFC circuit in Fig. 4. The PFC circuit has a $230 \mathrm{~V} / 50 \mathrm{~Hz}$ sinusoidal input voltage and a nonlinear load at the output. The peak-current controller has a switching frequency of $20 \mathrm{kHz}$. Both examples are simulated with the proposed method. For demonstration purposes, the event-driven method is executed with the classical fourth order Runge-Kutta method (RK4). Two event localization algorithms are applied: a linear interpolation algorithm (ED1) and a bisection root finding algorithm (ED2). The order of accuracy is compared with the backward Euler (BE) time-stepping method. In intervals without events, the Local Truncation Error (LTE) for a step of size $h$ is $O\left(h^{5}\right)$ for RK4 and $O\left(h^{2}\right)$ for BE. In time steps with an event, the local truncation error is $O(h)$ for BE or RK4 without event localization [2], $O\left(h^{2}\right)$ for RK4 with ED1, and $O\left(h^{5}\right)$ for RK4 with ED2.

The simulated inductor current is shown in Fig. 5a for example 1 and in Fig. 5b for example 2. The maximum LTE in the simulated time interval is plotted as a function of the step size $h$ in Fig. 5c (example 1) and Fig. 5d (example 2). The maximum global error is plotted in Fig. 5e and Fig. 5f. For example 1, the theoretical order of the integration method is 


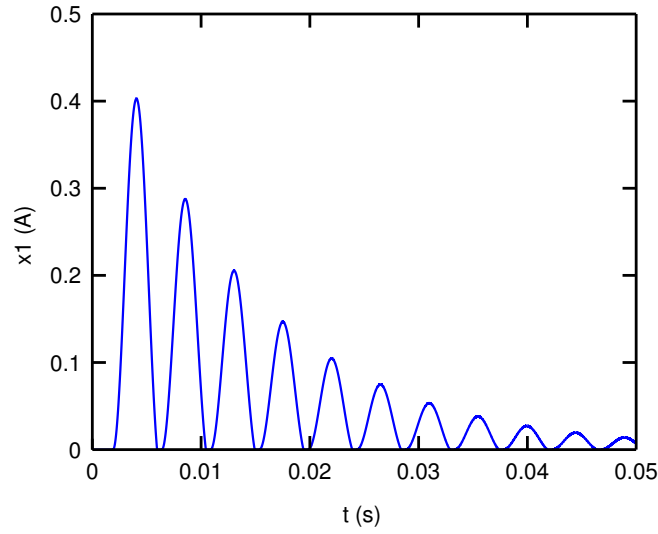

(a)

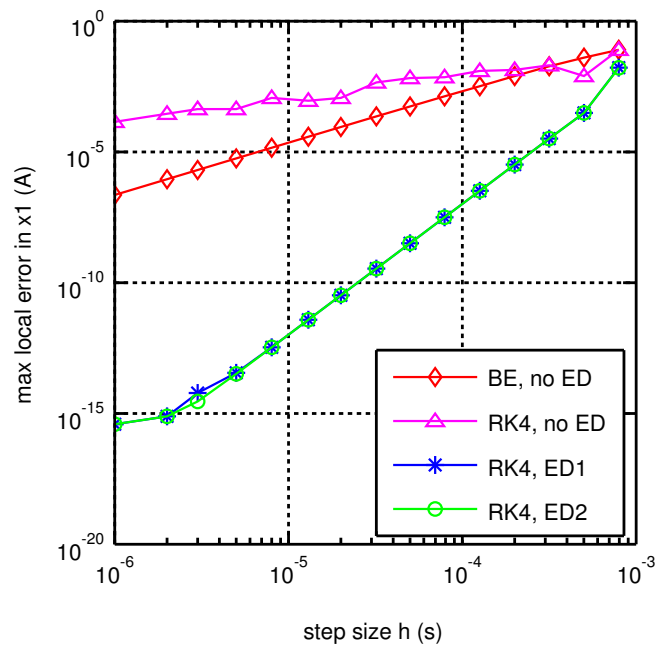

(c)

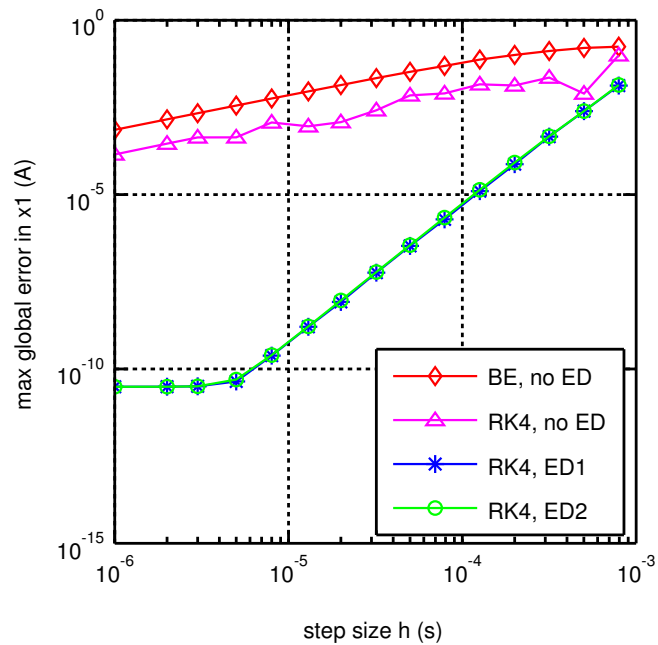

(e)

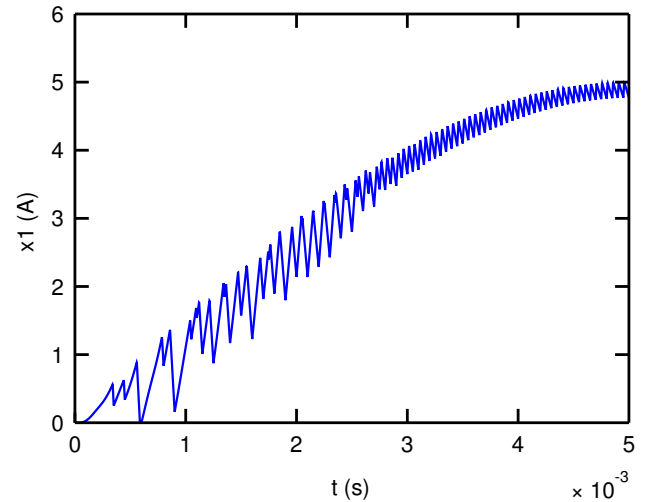

(b)

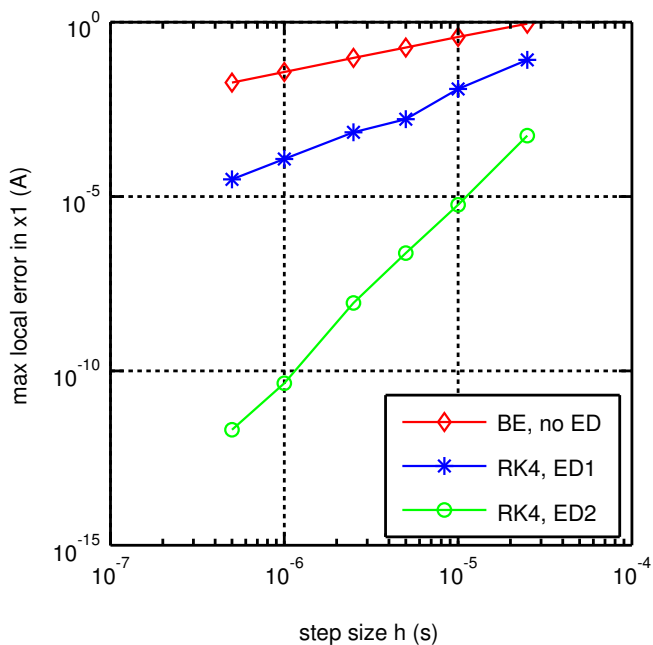

(d)

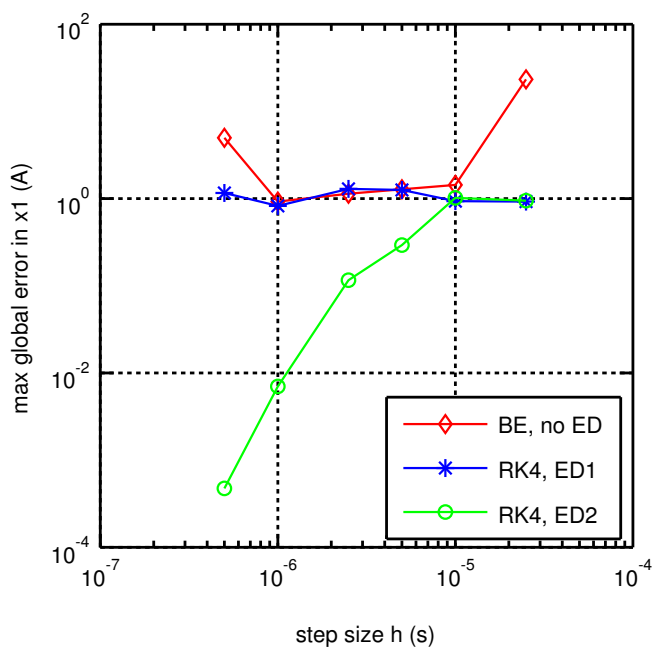

(f)

Fig. 5. Transient simulation result for the inductor current of numerical example 1 (a) and numerical example 2 (b). Maximum local truncation error and maximum global truncation error as a function of the step size: figures (c), (e) for example 1; figures (d), (f) for example 2. 
achieved, because the time step with maximum LTE is located in a smooth interval without events. In example 2, the smooth trajectories are less complicated: the inductor current is almost linear. The maximum LTE is therefore mainly determined by the event localization algorithm. The BE time-stepping LTE is only $O(h)$. The step size has to be reduced significantly to reach a reasonable accuracy with the BE time-stepping method, as compared to the RK4-ED2 $O\left(h^{5}\right)$ method.

\section{CONCLUSION}

This paper proposes an event-driven method as an alternative for time-stepping methods for the simulation of power electronic circuits in the complementarity systems framework. The main argument cited in literature for using the backward Euler time-stepping scheme instead of an event-driven scheme is its ability to advance the time step without event localization, and the ability to continue the simulation when there is an accumulation of events, for example in systems with sliding modes or Zeno behavior [10], [11], [17]. This behavior is unlikely to occur in practical control systems, as switching devices have a finite maximum switching frequency and behavior with infinite frequency should be avoided. The major disadvantage of time-stepping schemes is the low order of accuracy. The proposed event-driven method allows the usage of higher order integration schemes, resulting in a higher accuracy for the same step size. Furthermore, it is easy to integrate the control system in the event-driven scheme.

The presented numerical results demonstrate the importance of the event localization algorithm. The local truncation error of the event localization algorithm has to be at least of the same order as the integration method, to maintain the theoretical order of the integration method. Consequently, it is possible that multiple iterations are needed within one time step to find an accurate estimation of the event time. However, in modern DSP based PWM control systems, the switching time is already known at the start of each switching interval. In that case, only diode zero crossings have to be located.

\section{ACKNOWLEDGMENT}

J. Tant has a Ph.D. fellowship of the Research Foundation - Flanders (FWO).

\section{REFERENCES}

[1] F. Yuan and A. Opal, "Computer methods for switched circuits," IEEE Trans. Circuits Syst. I, vol. 50, no. 8, pp. 1013-1024, Aug. 2003

[2] P. Maffezzoni, L. Codecasa, and D. D'Amore, "Event-driven timedomain simulation of closed-loop switched circuits," IEEE Trans. Comput.-Aided Design Integr. Circuits Syst., vol. 25, no. 11, pp. 24132426, Nov. 2006.

[3] D. Bedrosian and J. Vlach, "Time-domain analysis of networks with internally controlled switches," IEEE Trans. Circuits Syst. I, vol. 39, no. 3, pp. 199-212, Mar. 1992.

[4] P. Pejovic and D. Maksimovic, "An algorithm for solving piecewiselinear networks that include elements with discontinuous characteristics," IEEE Trans. Circuits Syst. I, vol. 43, no. 6, pp. 453-460, 1996.

[5] J. Vlach and A. Opal, "Modern cad methods for analysis of switched networks," IEEE Trans. Circuits Syst. I, vol. 44, no. 8, pp. 759-762, 1997.

[6] A. Massarini, U. Reggiani, and K. Kazimierczuk, "Analysis of networks with ideal switches by state equations," IEEE Transactions on Circuits and Systems-I, vol. 44, no. 8, pp. 692-697, 1997.
[7] J. Alimeling and W. Hammer, "PLECS-piece-wise linear electrical circuit simulation for simulink," in Proc. IEEE 1999 Int. Conf. Power Electronics and Drive Systems, vol. 1, Jul. 1999.

[8] W. Heemels, M. Camlibel, and J. Schumacher, "On event-driven simulation of electrical circuits with ideal diodes," J. Européen des Systémes Automatisés, vol. 35, no. 4, pp. 467-488, 2001.

[9] V. Acary and B. Brogliato, Numerical Methods for Nonsmooth Dynamical Systems. Springer, 2008.

[10] V. Acary, O. Bonnefon, and B. Brogliato, "Time-stepping numerical simulation of switched circuits within the nonsmooth dynamical systems approach," IEEE Trans. Comput.-Aided Design Integr. Circuits Syst., vol. 29, no. 7, pp. 1042-1055, Jul. 2010.

[11] _ Nonsmooth Modeling and Simulation for Switched Circuits, ser. Lecture Notes in Electrical Engineering. Dordrecht: Springer Netherlands, 2011, vol. 69.

[12] A. van der Schaft and J. Schumacher, "Complementarity modeling of hybrid systems," IEEE Trans. Autom. Control, vol. 43, no. 4, pp. 483490, Apr. 1998.

[13] M. Camlibel, W. Heemels, A. van Der Schaft, and J. Schumacher, "Switched networks and complementarity," IEEE Trans. Circuits Syst. I, vol. 50, no. 8, pp. 1036-1046, Aug. 2003.

[14] C. Batlle, E. Fossas, I. Merillas, and A. Miralles, "Generalized discontinuous conduction modes in the complementarity formalism," IEEE Trans. Circuits Syst. II, vol. 52, no. 8, pp. 447-451, Aug. 2005.

[15] F. Vasca, L. Iannelli, M. K. Camlibel, and R. Frasca, "A new perspective for modeling power electronics converters: Complementarity framework," IEEE Trans. Power Electron., vol. 24, no. 2, pp. 456-468, Feb. 2009

[16] L. Iannelli, F. Vasca, and G. Angelone, "Computation of steady-state oscillations in power converters through complementarity," IEEE Trans. Circuits Syst. I, vol. 58, no. 99, pp. 1-1, 2011.

[17] M. Camlibel, W. Heemels, and J. Schumacher, "Consistency of a timestepping method for a class of piecewise-linear networks," IEEE Trans. Circuits Syst. I, vol. 49, no. 3, pp. 349-357, Mar. 2002.

[18] M. Ferris and J. Pang, "Engineering and economic applications of complementarity problems," SIAM Rev., vol. 39, no. 4, pp. 669-713, 1997.

[19] K. G. Murty, Linear complementarity, linear and nonlinear programming. Heldermann Verlag, 1988.

[20] S. C. Billups and K. G. Murty, "Complementarity problems," J. Computational and Applied Mathematics, vol. 124, no. 1-2, pp. 303-318, Dec. 2000.

[21] Q. Paris, "Multiple optimal solutions in quadratic programming models," Western Journal of Agricultural Economics, vol. 8, no. 2, pp. 141-154, 1983.

[22] S. C. Billups, S. P. Dirkse, and M. C. Ferris, "A comparison of large scale mixed complementarity problem solvers," Computational Optimization and Applications, vol. 7, pp. 3-25, 1997.

[23] S. P. Dirkse and M. C. Ferris, "The PATH solver: A non-monotone stabilization scheme for mixed complementarity problems," Optimization Methods and Software, vol. 5, pp. 123-156, 1995.

[24] R. Riaza, Differential-Algebraic Systems: Analytical Aspects and Circuit Applications. World Scientific, 2008.

[25] R. Frasca, M. Camlibel, I. Goknar, L. Iannelli, and F. Vasca, "Linear passive networks with ideal switches: Consistent initial conditions and state discontinuities," IEEE Trans. Circuits Syst. I, vol. 57, no. 99, p. 1, 2010. 